\title{
Utilizing Context in Location-Aware Short-Range Wireless Communication
}

\author{
Vesa A. Korhonen ${ }^{1}$ and Ritva Pyykkönen ${ }^{2}$ \\ ${ }^{1}$ Department of Mathematical Information Technology, University of Jyväskylä, P.O. Box 35 (Agora), 40014 Jyväskylä, Finland \\ ${ }^{2}$ School of Business and Services Management, JAMK University of Applied Sciences, Rajakatu 35, 40200 Jyväskylä, Finland
}

Correspondence should be addressed to Vesa A. Korhonen, vesa.a.korhonen@jyu.fi

Received 1 August 2009; Revised 30 October 2009; Accepted 25 January 2010

Academic Editor: Noël Crespi

Copyright (C 2010 V. A. Korhonen and R. Pyykkönen. This is an open access article distributed under the Creative Commons Attribution License, which permits unrestricted use, distribution, and reproduction in any medium, provided the original work is properly cited.

\begin{abstract}
We discuss how a short-range wireless communication service implemented for modern mobile communication devices can provide additional value for both the consumer and the service/product provider. When used as an information search tool, such systems allow services and products being promoted at the location they are available. For the customer, it may provide a "digitally augmented vision", an enhanced view to the current environment. With data filtering and search rules, this may provide a self-manageable context, where the user's own personal environment and preferences to the features available in the current surroundings cooperate with a direct connection to the web-based social media. A preliminary design for such service is provided. The conclusion is that the method can generate additional revenue to the company and please the customers' buying process. In addition to the marketing, the principles described here are also applicable to other forms of human interaction.
\end{abstract}

\section{Introduction}

Mobile computing and social networking are important trends on the early 21 st century in both private consumption and providing of services and products. Both of them have had an enormous effect to the ways the Internet services are being used. Less attention has been paid to the development of storage devices. Memory sticks can be carried anywhere, and especially physically very small memory cards have become an integral part of the mobile devices. A mobile handset may incorporate gigabytes of storage space due these devices. In addition to QWERTY-keyboard, there are highquality camera and voice recording capabilities available, allowing the creation of multimedia content with reasonable effort. Producing and sharing content while in nomadic move has become not only possible but also feasible.

While on the move, the users may also evaluate, comment, and discuss about virtually everything they happen to encounters including both products and services they purchase. Internet access from the mobile device is a key feature here. However, there are alternatives and additions emerging to the operator-oriented "traditional" wireless network access. Communication methods could vary depending on the location of the other party: being near, it would be possible to use Short-Range Wireless Networking (SRWN, for short), being far, the traditional Internet access is always an alternative. Quite similar to the way we used to have it without the mobile digital devices: friends near us could be visited or seen spontaneously, if known or assumed to be around. Friends overseas could be contacted via telephone, if one was there to answer it. Even if the media is completely new, the basic human behavior, with a strong locationdependency, has not changed.

However, what is changing is the production of content. The company-defined practice for promoting a service or a product is now completed with consumer-created view to the same matter. Users have the tools, the connections, and the forums for creating and maintaining online up-to-date view of the consumer. The company can no longer ignore the presence of such alternative truth, but it should cooperate with the consumers and listen carefully to what kind of events and opinions are taking place around their product. One could consider this process to form an "online dialogue" between the customer and the company. In this article, we further expand this process by introducing a connection 
between the near-surroundings-focused short-range communication and the location-independent, Internet-accessbased social media. We base our discussion on the theory of marketing, especially to the areas of marketing communication, segmentation, and branding. But we also believe the results will be applicable to much wider area of use of the SRWN. Applications on health care and education, among others, may benefit from similar models. However, due to the marketing approach of this presentation, we use the general terms "company" for the service provider/product seller and "customer" for the end-user purchasing the service/product. But these names should be considered to cover more than just what their basic business-related interpretations suggest.

This article is organized as follows: Section 2 describes the related work and technological background on the area, considering both the networking techniques and the principles of marketing. Section 3 further focuses on the marketing-related details, considering the needs of a customer and how they can be expressed and fulfilled by constructing a personal environment with the help of the mobile device. Section 4 summarizes some of the obvious challenges on the area. Section 5 provides a suggested application design for such system. Finally, Section 6 concludes the presentation.

\section{Related Work and Technology}

In this section, we introduce the key concepts and technologies which are used throughout this presentation. Both the short-range wireless networking techniques and the required background on the marketing are discussed here.

Our discussion is very much inspired by Beale's classical account of BlueDating [1]. It is an application which already contains several important aspects of the personal context discussed in this article. Providing one's own profile (perhaps with a photograph and even a recorded voice greeting) forms the basis for potential dates to choose whether or not to contact the sender. The invisibility of this kind of communication should be noted: If a receiver does not answer such "call", then the other party is not aware of one's presence, in the SRWN sense, at least. In other words, the user has the final control on performing communication or not. In this work, we generalize and further develop these fundamental properties for more business-like relationships, at the same time, we assume, without the loss of generality. Proximity of the communication parties is an important matter here: the two parties of the transaction must, more or less, encounter each other. For Beale's application, this is an advance, as it is for those services outlined in this article. However, as the proximity is combined with invisibility and anonymity, such form of communication may also allow it being misused for purposes such as anonymously delivering illegal content. The "Dark Side" of this technology, as we call it, may appear this way. But we consider such discussion to be beyond the scope of this article, so we only refer to articles $[2,3]$.

Another example of the social dimension of SRWN can be seen in the Nokia Sensor application (See http://www.about-nokia.com/blog/index.php?itemid=27), which provides services similar to the BlueDating system described above. Nokia Sensor provides also maintenance and search of the existing contacts. "A useful way to track down lost friends (or make new ones) in a crowded or dark spot", as the introduction notes. Here the "track down" part is important; the system must contain long-term memory for storing and maintaining the contact information.

In general, the SRWN technique may be defined to contain any wireless communication method which allows the communicating devices to exchange information without the involvement of operator or other administrative organization. Technologies such as Bluetooth (See https://www.bluetooth.org/apps/content/), WLAN (See http://www.wlan.org/), and ZigBee (See http://www.zigbee.org/) can be used for this purpose, depending on the required amount and type of content. For interactive and/or automatic SRWN applications, special software applications may be required. But since most of the intelligent hand-held devices provide built-in support for transferring simple data items (such as images) between devices using Bluetooth, it appears (and it is further discussed later in this article) that working solutions can even be built without requiring a special software support from the customer end.

A vital contribution to making the use of SRWN possible is the tendency to always having "on" the personal handheld data device(s) we are using. The field of these devices is so wide that we have adopted the name PMID (Personal Mobile Information Device, see [2]) to be used as a general concept describing the portable electronic communication devices we use for daily activities. A PMID can be anything from a simple voice call-and-SMS - only mobile phone to the area of mini laptops with G2 or G3 (wireless broadband) connections, which allows the user being contacted independent of his/her location. What these devices also have in common is the ability to include the personal content, the physical implementation allowing the device being carried with no much extra effort, and, at the same time, the ability to communicate even while moving. An important (but not fundamentally crucial, as it is shown in this article) factor is also the possibility to build and maintain taskspecific software for these devices. These devices allow us to collect material and resources to the device we carry with us, to within the environment we use for work and leisure, and even to both of them at the same time. The development has been quite apparent for some time, but the so-called Mini Laptop Phenomenon (described with more detail in [2]) has bought it to the mainstream, within the reach of any end-user. Smart phones have been attracted the more technically oriented people for around ten years, but within the last two years the much-PC-like handy minilaptop devices have gained new users from the less-technical members of our society. User interface following the desktopPC standard, easy connectivity to Internet and compact size have contributed to this.

Another class of services which have gained popularity within the last few years are services which allow combining data from different sources ("mash-up"), like embedding 
your own business ad to a map information viewable from everywhere have gained popularity. Google Maps (See http://maps.google.com/) is perhaps the most well know of such services. These are excellent utilities when planning one's route through a new city, for example. But when at the location, it may be difficult to browse the map with one's handheld device. In that case the SRWN-based services may provide assistance. Being able to create SRWN contact also indicates that the device is somewhere within the near surroundings. In other words, creating, maintaining, and dropping a communication link provide rudimentary location information for both the service provider and the customer. This kind of "micro GIS" service may also provide additional value for both the customer and the company.

Research concerning SRWN networking has been made under various titles. MANET (Mobile Ad-Hoc Networking) and VANET (Vehicular Ad-Hoc Networks) are perhaps the most often used of these. We base our discussion to the Mobile Encounter Network (MEN) model for SRWN services, emerged in the work of Kurhinen and Vuori [4] and has since been further developed by Korhonen and Kurhinen [5, 6] and Volovikov et al. [7], among others. Hsu and Helmy [8] and also Tanachaiwiwat and Helmy [9] have also considered encounters in WLAN networks with great detail. Walker [10] has also made important contribution on the area. Based on this accounts we are confident that networking via encounters is an important, developing area both in research and in end-user applications.

MEN networking is basically a matter of smart devices being able to proactively send and receive data packets while being within each other's communication range. No mobile operator support is required for this, but the SRWN is used for communication. Mobility of the devices provides the transfer medium: after receiving a data packet the device moves and, while encountering another device, may further distribute the data. The essential concept is the encounter event. This model allows different approaches taken to the communication. "Mobility", for example, may be a property of both or only one of the devices, or of an intermediate device, having no interest into data being transferred by itself, but just providing the transport service. Such diversity is the basis of the power of encounter networking.

Korhonen [3] summarized some key features of the technology, which are presented on Table 1. Those properties mentioned there define the limits an encounter networker is operating within. Locality, dictated by short range of communication, relatively small data size based on short duration of the encounters (due to mobility) and limited resources (such as PMID screen resolution and battery power) are the most important of these. Compared to [3] (where the full version of Table 1 originally appeared), there are two new properties being identified: the Sharp focus and Invisibility, which bore to the essence of this work. However, these features should not be seen as problems, but the MEN communication should be considered to provide a niche of its own.

Note the different but related functions and meaning of the three concepts considered in this work: a PMID is the end-user hardware and the interface to the system.
It possibly includes an operator-driven connection to the Internet. SRWN provides the technology for single-hop communication between different PMIDs. And finally, MEN (with the encounter approach) is the medium, formalizing the events allowing SRWN to take place.

In this work, we have chosen to examine the potential of SRWN on the area of marketing. To allow the reader to gain better insight into this process, we also need to introduce some basic concepts of marketing theory. In marketing communication model, a company formulates a message, uses different kind of media, and waits for the response (see Armstrong et al. [11]). The social dimension has been quite thin in this kind of transaction. More detailed and personal approaches have been developed by the companies, but these are highly cultural-specific. From the Nordic point of view, it is common to provide "club" or "society" membership for loyal customers (which status, in practice, may be gained by just one visit). The customer may receive small discounts via such membership, for example. At largest, it is possible to organize theatre events or trips at discount price. In spite of the simplicity of such operations, we still see here a clear social component in its infancy.

To further formalize the process of marketing, we should name several classical concepts which still serve quite well for the analysis of marketing as a communication. A model called AIDA (for Attention, Interest, Desire, Action) has been introduced already in the late 1800 s by Lewis (see Kotler et al. [12] , e.g.,). Despite its age, this model is still very useful for modeling the phases of customer experience. Especially it turns out that the digital augmentation of customer contact suits to this structure quite well. The "Attention" and also the "Action" phases can be enhanced via digital service.

Another key term to be considered here is segmentation. A company attempts to divide the heterogeneous markets into smaller segments to match the products and services more efficiency to the unique needs of different customers. (Again, see Armstrong et al. [11].) However, the concept of consumer segments has become less reliable. Customers, the consumers, belong to the multitude of segments. At the same time the new technologies give new digital tools for interacting with other targeted customers [11]. These members of the so-called online population, the iCitizens, are creating and sharing content, and using it for influencing each others. They are new experts and tastemakers trusting each others' buying experiences in making their own buying decisions (refer to Mooney and Rollins [13]). It should be noted that this also allows misbehaving members of the community to cause confusion, that is, the new model of decision making may even be more vulnerable than the traditional approach (the "Dark Side", as referred above). Clearly, however, online customers are gaining more control of any marketing activity. The Resource Interactive 2007 iCitizen Motivational Study already stated that $86 \%$ of the U.S online population (200 million people) were creating, sharing, and influencing each other (Mooney and Rollins [13]).

The goal of a company is to create products and services which differ from their competitors sufficiently enough to be identified as products of just that particular company. 
TABLE 1: MEN key properties (subset from [3]).

\begin{tabular}{ll}
\hline Property & Notes \\
\hline Small data size & $1-100$ kilobytes. (Short duration of encounter connections.) \\
Low security & Public data. (Compare to hand-out flyers.) \\
Low dependability and low value & The user may do almost as well without it. (No mission-critical information.) \\
Locality & The usefulness of the information has high dependency on the current location. \\
Short life-cycle & Information may be valid only from minutes to days. \\
Faster distribution & Even the fastest direct marketing is slower. \\
Sharp focus & Combination of locality and speed of the communication. \\
Invisibility & Wireless connection does not give indications of the other user(s) presence or exact location.
\end{tabular}

In other words, the company may wish a product or set of products or services to appear as associated, easy-identifiable family, a brand. Keller [14] describes this process as four steps of brand building. On the first step, the company must ensure the identification of the brand with customers and a brand association in consumer's minds with a specific product/service class or customer need. On the second step, the target is to establish a totality of brand meaning in the minds of customers by linking a host brand associations with certain properties (such as colors, logos, and other visual cues). On the third step, the company must elicit the proper customer response to this brand identification. And on the fourth, final step, the company must convert brand response to create an intense, active relationship between customers and the brand [14]. Hence the brand has a context in itself, where the customer moves and makes decisions. According to Mooney and Rollins [13] , the social media actually helps marketing communication to move away from the single consumers brand experience. They discuss about concepts on-demand experience, personal experience, engaging experience, and networked experience. An open brand strategic framework is defined in [12], indicating both the social web's traits and technologies and the citizenry's power to be both medium and message.

The concept of self-segmentation has been suggested to being form. Online companies are building correlation clusters between purchased products and using the cluster information as recommendations. Self-acclaimed interests of products and services will be shared in the social network Facebook (http://www.facebook.com/), Twitter (http://twitter.com/), LinkedIn (http://www.linkedin.com/), and other social media services. Consumers can use a dealalert function that automatically informs consumers about something new or interesting in the "opted-in" interest domain of a consumer (such as Google Alerts (http://www .google.com/alerts), Orbitz (http://www.orbitz.com/) and Expedia Fare Alert (http://www.expedia.com/daily/highlights/fare_alert/default.asp), e.g.,). Introducing the SRWN mobile tools, it is possible to further expand this, from the online companies and customers to their on-the-street counterparts.

More recent and information technology_oriented view to the "turning the customers head our way" has been discussed under the title of persuasive technology (see [15, 16]). As the name indicates, that area of research studies the use of devices (such as PMIDs with SRWN) and software (such as service provider's electronic material) for persuading the user (customer) to change his or her behavior. We believe that this area of research may provide important contribution to promoting the SRWN technology as it is described here. When compared to our earlier account [2], we see that persuasive technology may provide goals or targets for the user, which brings positive experiences to the customer and hence helps him or her to make a decision about using the service.

\section{The User As the Customer}

Our starting point in this study is customer-oriented: wherever the customer goes, he or she should find the services and products he or she is looking for without extra effort. Or, in the other case, the customer should be able to see that the requested goods may not be found on this area. Time is another dimension to be considered: an interesting event or special offer can be available only at some place only for certain time. Being able to benefit from that the customer should be at the right location at the right time, and further have the vision (or any other sense) allowing to note the existence of the offer. So considering also the time factor, one should have immediate access to the current information concerning that particular environment.

On some locations, such as shopping malls, the available sortiment and hence amount of information may be very large. The customer should hence have a method for expressing what particularly is important to him or her. There may be available numerous services and offers the customer wishes not to be aware of, but he or she would like to focus on those items which are significant at that particular place at that time. In this section, we discuss these three components, development of the user role, immediate access of services and the location-dependency. We believe that this customer-orientation also serves the service provider best. It is the customer, after all, who should be in the focus of operations.

3.1. The Development of the User/Customer Role. The more active role provided to the customer-user by the new technology may radically change the balance between the company and the customer. It is still unclear what kind of 
movement we are encountering. Based on the known history of computing it appears safe to say that the development will not be straightforward, but there is going to be various changes of direction within the development to appear. One cannot estimate whether the result actually increases the amount and quality of the social content, but leads to a more confusing and unclear marsh of disinformation. However, the history of the Internet clearly demonstrates that there is no return to proprietary systems for public information exchange. The solution for both the company and the customer is to adopt to the use of the modern communication channels.

Today's customer is more ready for the event of purchasing a product or a service. He or she has already queried information about the company, via the official company website and also via the unofficial, social media services considering the particular company, product, or service. The company has to understand the change and be able to refresh the concept of segmentation to self-segmentation. A company controlled brand building changes to open, customer controlled brand building, performed via the online dialogue between the company and the customers. The company must accept that it no longer has the full control and it no longer sets the rules how its products and services are exposed to the public discussion. The opportunity here is the possibility to allow personal relationships to form via the customers, allowing the necessary resources and skills being available on the company side. This could provide even more enhanced user experience and hence strengthen the loyalty of the customer.

One should compare this development to the current "battle" on the music/movie industry: the production companies, which are trying to maintain their old business model instead of trying to develop new business models, are eager to limit the Internet access of their products. So far this process has been justified by the current legislation, which provides the framework for operating within the global open-access Internet media environment. But considering the marketing of a product, the customers have much more power in setting the market the way they like it to be. Another, secondary goal of our work is to initiate discussion also on this area.

\subsection{Creating and Maintaining the Personal Environment.} The significance of the personal environment as a stepstone for a wider area of information services should not be underestimated. Considering the suggested personalized context formed using a PMID, we are already active users of such features. For example, we may have specialized ringtones for different calls we are receiving. The phone directory may also allow the image of the caller being associated with the phone number, so incoming calls literally display the face of the caller. Sometimes it is even heard that somebody have recorded and used his or her child's voice as a ringtone of a cell phone. This gives the phone owner a very useful and immediate piece of information of the potential urgency of the call and also prepares the phone owner for the right mood for answering the call. Even if bothered by the ongoing work-related matters, one is supposed to take the role of a mother/father when talking to one's child. This is a very simple but at the same time very clear example of creating context with your mobile device. The context is brought to you when you need it, and it helps you to perform the required task. However, since it only applies to incoming calls, it remains a quite passive method. But when compared to Beale [1], one can see that this is an addition to the distribution of dating profile data: instead of receiving data from other people created by the other people, you yourself have the full power to define how the other peoples' incoming calls and messages appear in your context. Hence on the existing human interactions, we already find personal preferences being used on both the sending and the receiving end.

More active context creation can naturally be applied. Filtering, (see Korhonen [3] and also Boström et al. [17]), is another basic feature for building a personal environment via limiting the incoming messages. If a service may "push" data, the customer is very likely to dislike those parts of the services which are of no interest to him or her. Some services may even be quite offending to some persons, so filtering them out may be the default value. Even the detailed technical specification of such services is beyond the scope of this presentation; we must note that this also sets strict requirements to the implementation of the filtering: if the customer absolutely wants to skip services attributed with certain keywords then it should not be necessary for him or her to manually enter these phrases to be avoided. But the filtering should stay within the same positive personal context the whole use of the device is. A solution of specifying only those phrases which are allowed to pass through is not a good practice, since, despite being too laborious (leading to the ignorance of the use of the service) it also prevents the controlled augmentation of the context discussed below. The idea behind effective filtering lies on the unofficial role of SRWN networking: If the customer sets up a PMID for receiving SRWN data, he or she may also choose to reject any of those data items he or she is not interested in. With the traditional mobile services (voice call, SMS), this is not usually done automatically (even if you may manually reject a disturbing incoming call). For SWRN such traditions of "always answering" do not exist, allowing the effective use of automatic services in filtering. The augmentation of the personal context may take place by allowing certain phrases (such as "new" or "only today") to pass the filter independent on their full content (but still considering the phrases specified to be offending, of course). Hence the result can be a personal environment specifying the personal needs and interests, but which still is active and can be allowed to augment within specified limits. Gaining the attention of such customer is hence possible and using the SRWN media will therefore appear interesting to the companies providing their products and services.

Above we emphasized the significance of context in this area. As a further development of the mentioned active and passive environments, we may also see here a strong context and a weak context, depending on whether the service or the user is setting the limits of combining the local 
input to the personal preferences. However, the intermediate form, dynamic context, may be the goal to be achieved. The service should be available everywhere, but the user must have the feeling of being in command. Searching the Internet, as it is commonly done now when looking for a certain service or product, has no personal context (at least strong one) associated with it. Obviously this, too, is already changing: Web services, for example, may give you "personal recommendations" based on your earlier actions. But these personalized features remain within your digital window to the world (your PC screen), they are not following the contents of your immediate surroundings. So it seems reasonable to reserve the "low context" category for these "advanced traditional” web services.

3.3. Promoting and Discovering the Services. As suggested above, for someone willing to reach a PMID user, such as a company marketing a product or providing a service, it would be very beneficial to be able to get within the already personalized context of a potential customer. Even with the introduction of electronic services in the Internet, there still exist large amount of business where each bypasser of the service provider's quarters is an important or even the most important potential customer. SRWN, the technology behind this discussion, allows these people being reached, even with a strong dependence on the location. The technology brings to the company the first interaction with potential customers and it can be also a start for the customer collaboration and the dialogue, start for the customer relationship (see Peppers and Rogers [18]). But the huge difference lies in the fact that in our view it is the customer who has the final control. When considering the significance of "personal" with more detail, the following appears reasonable: the mobile device user may like to see what is around her or him, but most likely does not wish the environment to get too close. This is a matter of finding a balance between the "pull" access (being able to search the internet with specific keywords) and "push" access (being given large amounts of information automatically to your PMID) without disturbing the user. On the other hand, for the service provider being found via Internet search is a long shot: there are myriad of others providing similar service. More or less the same applies to business catalogs in printed form. It is easy to get lost in the mass. However, promoting one's presence for potential customers already in the same area is definitely more focused way of advertising. And the task of focusing is mostly left to the customer. One can say that, from the customers' point of view, the PMID is providing an enhanced way to see things. It is forming a new sense to us, a digitally augmented vision (cf. to Scott et al. [19]). It is companies responsibility to not to "ruin this vision" with too ardent broadcasting of messages.

From the service providers' point of view, the situation is straightforward: they would like to know how they can serve the customer better. What are one's main interests, what he or she is looking for? This turns to the problem of knowing the keywords the customer has included in his filtering system. In that task, traditional branding and segmentation may still provide support for finding the right terms, as also does the active participation in the on-line dialogue between the customers: knowing how the product or service is handled in the social media allows the company to direct its campaign to the right direction.

What is the material the service provider is using for promoting services? Basically, an electronic business card can do most of the job, indicating the company's name and services provided, completed with contact information. More advanced version may appear much like the web page of the company, including availability and pricing of the product or service. The information may also contain offers which are valid only for limited time. Or the number of copies of the message to be distributed may be limited. The technology behind distributing the data may be mobile widgets (see $[20,21]$ ), small application-like pieces of code which are specialized to one task. Providing the ability to receive SRWN product promoting messages and react to them is a suitable task for a widget. Later, in Section 5, we further examine the technological side of promoting the service.

At this point, we like to summarize that, based on the discussion above, SRWN services using encounter networking may obviously support the marketing communication and segmentation/brand building-related tasks of the service provider. What remains to show is how benefit may be gained on the customer side.

3.4. The Immediate Access. We argue that proximity is essential to the immediate access of a service. However, the immediateness should apply both to adopting the service and utilizing the content it provides.

Considering the use of SRWN as a part of marketing mechanism, the short range of the communication should not be seen as a limitation, but as an opportunity. It brings a new dimension to the networking: a local context. This is invoked by the "micro GIS" property discussed above. Consider a busy business street with many types of services and many service providers for them. Each of them may provide their contact information via business catalogs and web pages. But it still may be quite hard to reach the customer just a few feet from the entrance. In our work, we assume that using short-range networking, the customer may easier recognize those services and products which are near him or her at this moment. This allows him or her to make more rapid decisions about his or her further movements. And conversely, using SRWN, the service/product provider is able to gain the attention of those potential customers who are close to his facilities and hence are most likely to drop in within the near future. Such ability introduces the concept of locality to the mentioned on-line dialogue between the parties.

Information received via encounters may actually be a (set of) $\operatorname{link}(\mathrm{s})$ to other services and resources. The PMID is a smart and relatively fast device; it can do the work of receiving input and formatting it for display (and even for sharing), based on a customer selfdefined profile. Here we have an enormous potential for 
the company to be utilized. During operation, it would even be possible to combine data received via SRWN and via other methods, such as visual clues. Point the mobile phone camera to an image tag, such as a printed matrix code, to receive input, for example (A Finnish playgrounds equipment manufacturing company, Lapp-Set, is already providing such feature as a part of a mobile game platform, see http://www.tekniikkatalous.fi/ict/article299580.ece (in Finnish)) . And if it would be possible to use RFID tags, there would even be no need to point the phone. Direction/movement-aware devices (with GPS support) could further enhance this service.

As also noted in [2], MEN-based communication is "local, low-value short time-frame open information exchange in action", and without exposing neither of the parties to extensive security threats: considering the basic unit of MEN communication, a single data item (a packet transferred within one encounter), this basic block has a very low value as it is. It requires the context where it is to be applied to have greater value. There is a direct analogy to leaflets and flyers being distributed on the street, except SRWN-based communication being more economical, more ecological, and more focused.

One can even imagine this augmented context forming an implementation of the discussed self-segmentation of the customer: the seller just pushes all the information he can, and the receiver(s) take care of filtering it by their personal needs. Consider the AIDA model: the "Attention" is obviously a necessary element for the whole chain to become active. Here personal, hand-held mobile devices may turn out to be very valuable. SRWN can hence be an effective way to do not only one-to-many marketing but also one-to-one marketing, perhaps also many-to-one. Actually the cardinality of the information distribution is no longer controlled by the service provider, but the customer, each for their own respect. "Action", the last part of the process, may also be enhanced using the SRWN support.

\section{Challenges}

There are several obvious challenges in the SRWN-marketing system outline described above. This section combines those items and provides a preliminary discussion about the severity of the issues.

Security issues associated with the augmented personal context are definitely worth discussing. The biggest threats here are physical: having your device stolen can be catastrophic. Sever loss of data may occur if you have not applied appropriate backup conventions. With mobile devices (from tiny MP3 players to full-size laptop computers), this has been true for decades. Hence there already exist a culture of taking care of your device and creating backup of the important information contained in them. For laptop computers also data encryption has become a standard procedure. Based on this, we assume that the physical security can reach reasonable level with no new requirements set to the enduser.
Considering the network security, an SRWN model appears even more secure than traditional Internet-based networking. Cookies, pieces of information, which can be used to provide personalized web experience, may also allow the user actions being monitored and traced. The context, when browsing the web, is not completely controlled by the end-user. The MEN networking discussed here provides more secure method for gaining the same results. It is true that the MAC id of a mobile device may be recorded by the server and such property may even allow the customer's daily whereabouts being monitored. However, assigning a certain SRWN MAC address of a mobile device to a person is not as straightforward as in traditional, operator-driven Internet access. And the filtering taking place within the PMID is completely invisible to the service provider, giving no indication to the sender whether or not the posted message is actually appearing on the customer's screen. Hence in SRWN-based system, the personal context remains true personal. However, as more advanced applications are introduced, it is obvious that some forms of malware will be distributed "on the streets" via SRWN. A form of "sandbox" implementation may provide solution to this problem. An area of future research is how to develop the filtering process to automatically detect and ignore any incoming message which appears to be harmful for the receiver.

A fast movement is definitely an area where the SRWN communication can be difficult. In Vehicle-to-Vehicle (V2V) or Vehicle-to-Roadside (V2R) connections, the time within each others' range may be so short that the data transfer does not succeed or remains very limited. Here context networking is mostly unidirectional, performs better in this sense. But using other communication methods in addition to SRWN, such as RFID or visual tags (see [2]) may also partially help to solve the problem. The smaller amount of content within these methods of data exchange may be compensated by the Internet connection available virtually on any device of MEN use: receiving a web link (or even only part of it, to be appended to domain information received when entering the area) may allow equal building of augmented context. However, naturally the (wireless) Internet connections of a popular location may also suffer from lack of resources.

Considering a popular, crowded place, the SHWN channels may be overloaded. Due to the short-rangeness, such overloading remains local (cf. also to Table 1 ). In overall, since based on the diffusion-oriented data transfer approach, encounter networking systems are very scalable: no centralized server could go down and completely deny the access to the service. If there is a local overloading in the network, it remains local. All the other parts of the network are still operating. This also applies to the appearance (and disappearance) of new service providers: a new company may join (or leave) the network without the need to register or perform any other administrative tasks. As noted in [3], encounter networks are not created or built, but they emerge.

Considering potential overhearing or even eavesdropping, the unit of SRWN-encounter communication, a single data item has a very low value as it is. It requires the context where it is to be applied to have greater value. Also public, 
nonmission critical data contents actually make overhearing a desired event (more effective marketing) and at the same time make eavesdropping an absurd term in this context. There is, again, a direct analogy to leaflets and flyers being distributed on the street.

As pointed out in [3], no mission-critical data should be sent. There are other, more effective methods for crisis communication, for example. Consider an emergency, a fire in a shopping mall. There are traditional methods for informing (alarm bells) and protecting (fire extinguishers, exit signs with emergency lighting) those under immediate threat and more modern methods (area-specific SMS) to inform the others to avoid the area. At that situation, the SRWN communication becomes obsolete and should be ignored to provide space for more effective and secure messaging.

Overall conclusion concerning the effect of challenges on encounter-based SRWN communication lies on the phrase "augmented". The SRWN augmented context is not the whole, but an extra plus on the personal view to the current environment. Especially SRWN is not for protecting life and property. One should compare the system described here to those used for direct marketing: if one, for some reason, misses a flyer being distributed, he or she may be angry but there is no damage caused to either of the parties.

\section{Application Considerations}

This section concentrates on the implementation details of an SRWN-based encounter networking utility. A preliminary application test is described. The required software components and their deployment are also discussed.

5.1. Accessing the Service. One critical item in utilizing SRWN is how to set-up the users' PMID for some particular environment. Having a common system obviously requires some cooperation from the area's service providers. "Area" here may stand for a city, a business street, or a shopping mall, for example. A common method and technology should be defined and implemented. As a piece of this process a PMID software (if required) should be born, together with the instructions how to retrieve the software. Maintenance of the service should also be considered. In MEN type systems, however, this is quite straightforward. Providing content should be made possible for an individual service provider. But since there is no server or network to be maintained, there is no actual electrical infrastructure requiring resources. Informing the customer how to join the system and how to access the necessary software must be kept up to date.

A natural solution would be to implement the required special software for some of the most common platforms (S60, Windows, Linux) and allow it to be downloaded from the internet. Physical media (billboards, signs, flyers) or homepage of the environment (if applicable) could then be used to distribute the address for downloading. A more advanced version could also provide ready-made filters for different customer profiles, based on age, gender, hobbies,

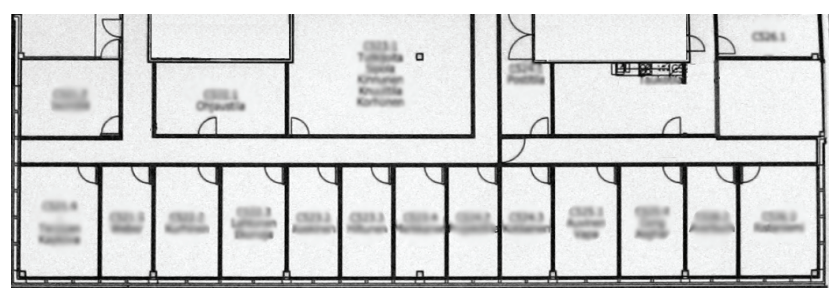

FIGURE 1: Sample map image for AutoHelpdesk application.

and other interests. This level of service already provides most of the more focused access based on the current location of the user.

For the simple version the data item being transferred may be a single image file (as on the application test described below). For more active content, the data may be in the form of XHTML code, including images. Such packet is standard, browsable, and allows linking to the public Internet. Using mobile widgets [17] is a technology which appears to be suitable for this purpose.

The scalability of MEN networking allows the system to grow and to reduce without special effort. New service providers may open and discontinue the service as they like. In general, SRWN-based solutions appear to be technically less demanding when directly compared to Internet-based marketing. If it wishes to do so, the company may run the system with very small amount of resources, repeating the same advertising message from day to day. However, it is also possible to fine-tune the company side. What keywords should be present to catch more attention, for example.

5.2. A Simple Application. Consider a very simple application, which could be used as a starting point: an automatic helpdesk attempting to send a map of the area (as an image) to any other Bluetooth device it discovers is a very basic but still useful application. No specific action, in addition to activating the Bluetooth, is required by the end-user. This may take place by a sign informing the user with an appropriate message. Also a notification about the security risks related to this should be included. For example, the user should deactivate the Bluetooth connection after leaving the area. A recommendation to not to accept inputs from other sources than that specified in the documentation should also be made.

A preliminary test of the AutoHelpdesk application was made using manual procedure: a Nokia N810 device was loaded with a $1036 \times 347$ pixel grayscale map image (Figure 1) containing information of the seating order of an office block. The image file had a size of 223 kilobytes, which appears reasonable for Bluetooth transfer but still allows a resolution high enough to be easy to read. (Note: for security and privacy reasons, the image shown here has been blurred to hide the exact name and location information of the persons involved.) An instruction sign was created (see Table 2) providing the necessary information.

In the first demonstrations sending the image map was done manually: it was first agreed with the other party 
TABLE 2: Instructions for the AutoHelpdesk service.

Bluetooth AutoHelpdesk available

To receive the map of the area, please, activate the Bluetooth service of your mobile device.

A device called "AutoHelpdesk" will shortly attempt to send you an image. Please accept the transmission to receive the map.

As a security precaution, DO NOT accept incoming data from other sources than "AutoHelpdesk". You should also consider deactivating your mobile device's Bluetooth after receiving the map.

when the transaction should take place. When the receiver was ready, the operator of the N810 tablet activated the map file sending using the tablet's user interface. There were three mobile devices, Nokia phones 6103, 6200, and 6800 , used as receivers of the information. Up to 4 or 5 transmission transactions were performed with each device. From a distance up to 5 meters, the transmissions were successful. Above that, the results varied. However, no clear connection between the phone model and the success of the attempt could be made.

Even this scheme is somewhat oversimplified and too laborious for practical use (requiring constant attention, which is quite the opposite to what was the original idea of "automatic" helpdesk); it already demonstrated some properties of the system which should be considered when creating more advanced applications. The following three observations were made during the tests.

First, simple devices like 6103 may have its memory filled so near to capacity that even a relatively small file cannot be received due to lack of memory. This can be very frustrating to the user. To free space, one should browse through the Gallery and remove several of the existing images (which typically are of size from 30 kilobytes to 80 kilobytes). Such procedure is not feasible in the intended user scenario of the system. Obviously the instructions for the use of the service must include the space requirement for the data. However, with such requirement the part of the intended simplicity of the process is lost.

Second, it appears that activating the Bluetooth connection is not as straightforward as even a skilled phone user thought it would be. A need to "reboot" a phone was encountered. The range of a successful transmission also varied too much to allow the practical use of the system. Especially in this case the connection was established from the distance of approximately 15 meters at maximum, which is less than half of the diameter shown on the map image. We assume that a PC-based server with a Bluetooth interface would provide larger and steadier range than N810. We will organize further tests with such environment.

Third, how to fix one's location on the map received turned out to be a problem. On a large office building, there are several different ways to approach a certain point. When, independent of the direction, the same map is received, it may be hard to the receiver to find out which way he or she should read the map. On the test the map used displayed a relatively small area of the building. We believe this problem could be solved satisfactorily by using a map covering geographically larger area (several times larger than the range of the transmission) where the location of the AutoHelpdesk server could be marked. The exact positioning of the server should then be considered carefully. Also including such features as the main entrance and the main hall of a building can help the user to read the map.

However, the typical problem in SRWN, losing connection due to mobility, is less severe here. User, interested in a service, is likely to stop and wait for the information to arrive. The given instructions may even guide the user to this direction. Even attracting the user with a brief "teaser" and then sending more detailed advertisement for those who have chosen to stay in the range could be done in more advanced versions of the service.

The conclusion of this test was that there are challenges in implementing an automatic help desk of this form, but the overall concept appears quite promising. We will continue developing this application to more automatic versions.

5.3. Modeling the Application. Based on analyzing the simple application described above, it is possible to create a more general-purpose model. Below there are two components of UML model of a more advanced application. In this preliminary study, the models belong to the "analysis" phase of the design process.

Figure 2 presents a collaboration diagram illustrating the actors and other entities required. The flow of communication within the system is also presented. There are clearly two actors, the service provider (: ServiceProvider) and the customer (: Customer). Both of them have their specific user interfaces. A single control object, BroadcastEngine, may handle the process. For message storage, an AdvertisementBase data entity is used.

The flow of operation begins from the : ServiceProvider actor, who is required to initiate the application by setting up the service. The Message here refers to the data to be sent to the Customer. After that the BroadcastEngine is running and, when discovers a: Customer, fetches the data item from the storage and attempts to deliver it to the : Customer actor. Operation continues until the : ServiceProvider terminates the BroadcastEngine.

Figure 3 contains another UML model, the deployment diagram. This diagram illustrates the possible deployment of the different hardware and software components. In our sample application the configuring device and the server device were a single entity (the N810). But for more general solutions it would be possible to separate the configuration device, ConfigDevice. In its most simple form, this could be any Bluetooth device which contains a certain, predefined name which is recognized by the ServiceDevice, allowing new content being updated to AdvertisementBase. 


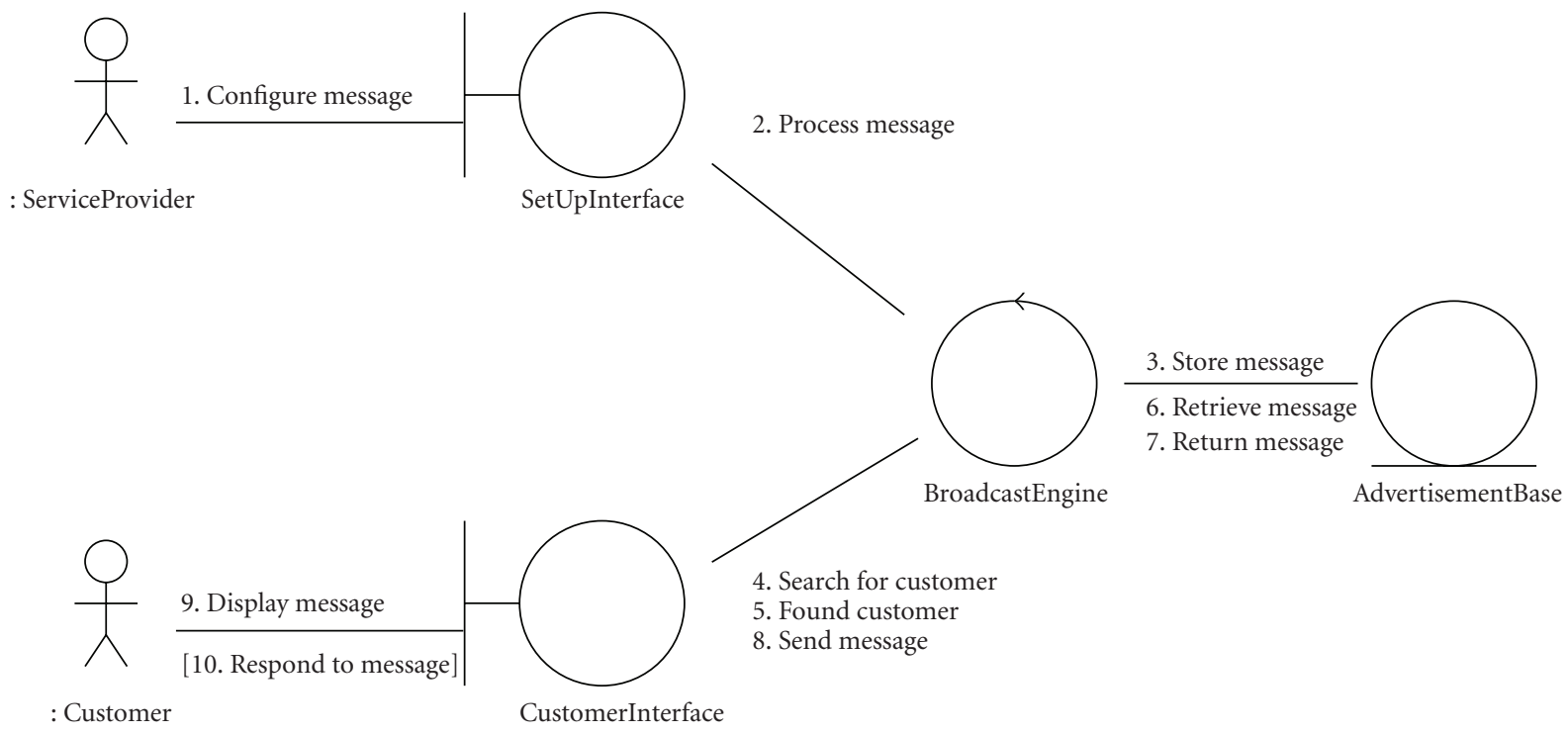

Figure 2: Collaboration diagram.

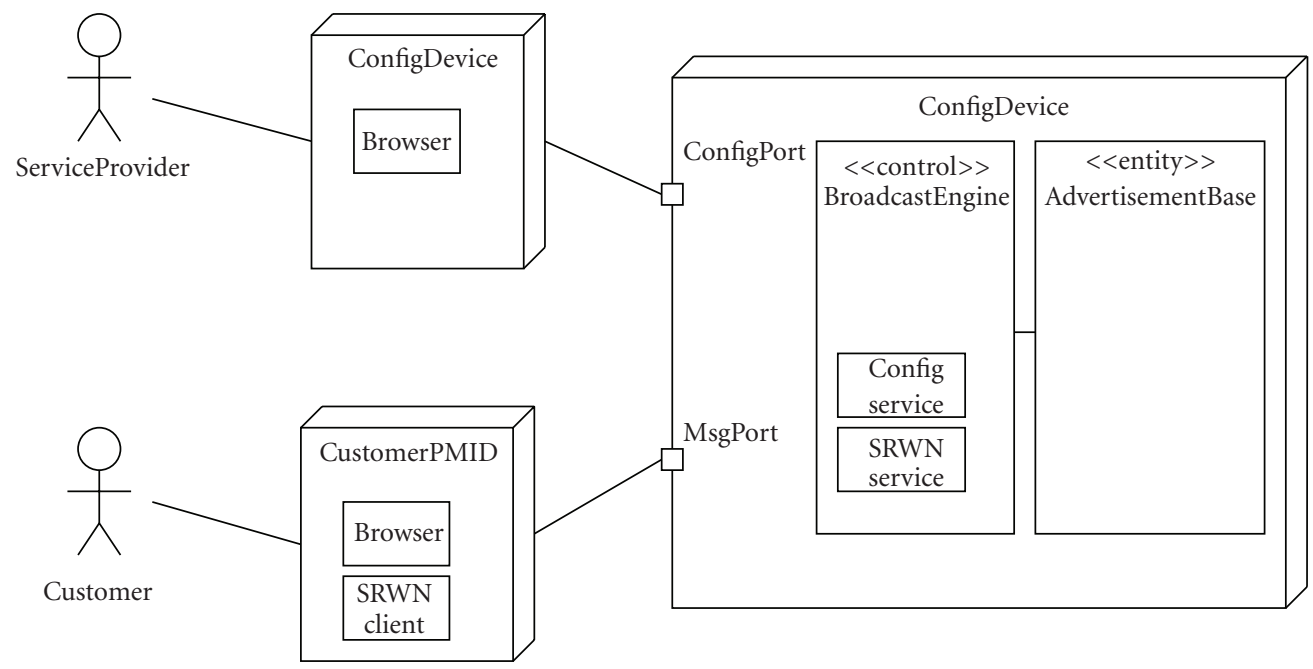

Figure 3: Deployment Diagram.

As shown on Figure 2, we assumed that a browser of some form is a key element in both maintaining and using the message to be delivered. Independent of the actual hardware platform, a browser is likely to be available on the target device, so such approach appears to be reasonable.

Even being relatively simple (due to omission of most of the technical details, which would be introduced in the "design" phase of the modeling), we believe that Figures 2 and 3 may serve as a starting point for a future work in creating a more detailed model of the application.

\section{Conclusions}

Personal context is an important part of mobile computing. Wherever you go with your PMID, you have both your personal environment and the local environment at your fingertips. Being able to combine these two worlds can provide better service quality and reduce the overall costs for both the customer and for the service providers on the area. The SRWN-based services (such as MEN) allow the user to gain an enhanced view to the current surroundings. And with Internet connection, they combine the current whereabouts with the global sharing of experience. Shortrange wireless encounter networking hence forms a "missing link" between the personal and the local.

It has been shown above that SRWN enables an ongoing information search on the market environment. It further supports the change of the traditional company driven massmarketing push tactic to the pull of online customers. This is a new media for marketing communication. A mobile device may initiate the company-customer dialogue much 
more effectively by providing a focused and unobstructed entry to the Attention phase of the AIDA model. Having a sign up on the side of the street promoting the service is easy to miss even if the customer is looking just for that service. And as the users will realize the benefits of saving time and money they are very likely to adopt the service to continuous use. This is the "controlled pull" of new customers, a creation of a dynamic context between the personal and the local environments.

The company has to refresh the concept of segmentation to self-segmentation. SRWN also gives the consumers new, more immediate tools to get involved in brand messages and service offerings. Combined with social web applications, it help brands to move away from the single consumers brand experience. From the users' (customers') point of view, one can conclude that the introduction of contextspecific communication makes the social networking more approachable: you may have immediate, more focused interaction within the surroundings, combined with Internetbased connections to those far away.

Considering the design of the system required for SRWNencounter communication, the main feature is its simplicity. The conclusion of this discussion is that encounter-based SRWN allows you to receive information because of where you are and who (or what) you meet, not because of who you are, even though your personal interests may allow you to filter incoming data. In a dynamic environment (with human-scale time limits) where the information is born in somewhat unexpected way, this is a good property. Here we also enter the area of ubiquitous computing: wherever the customer goes, the essential information is ready via encounters, instead of searching the Internet for some keywords likely not to be the correct ones. This idea very much provided the inspiration to the work considered in this article.

Our future work on the area concentrates on several issues: we look for methods how to augment a closed, personal context, in order to provide more room for the augmentation of the personal view. We assume this can be made with developing the filtering process. Malware detection is another matter the filtering system should consider. Another part of future work is also the inclusion of more detailed geographical information, replacing the "microGIS" discussed here with a real GIS information provided by a GPS device. Continuing the implementation of the outlined application (supported with the further development of the UML model) provides the background for examining these issues.

\section{References}

[1] R. Beale, "Supporting social interaction with smart phones," IEEE Pervasive Computing, vol. 4, no. 2, pp. 35-41, 2005.

[2] V. A. Korhonen and R. Pyykkönen, "Creating context as you go," in Proceedings of the 13th International Academic MindTrek Conference: Everyday Life in the Ubiquitous Era (MindTrek'09), pp. 37-40, ACM, Tampere, Finland, September-October 2009.

[3] V. A. Korhonen, "Mobile encounter network-the missing data link," in Proceedings of the 12th International MindTrek
Conference: Entertainment and Media in the Ubiquitous Era (MindTrek '08), pp. 80-84, ACM, Tampere, Finland, October 2008.

[4] J. Kurhinen and J. Vuori, "MP2P network as an information diffusion channel," in Proceedings of the 62nd IEEE Vehicular Technology Conference (VTC '05), Dallas, Tex, USA, 2005.

[5] V. A. Korhonen and J. Kurhinen, "Information exchange in mobile encounter network," in Proceedings of the $3 \mathrm{rd}$ International Conference on Networking and Services (ICNS '07), 2007.

[6] V. A. Korhonen and J. Kurhinen, "Logistic model for modeling mobile encounter network," in Proceedings of the 5th ITI International Conference on Information and Communications Technology (ICICT '07), pp. 151-155, Cairo, Egypt, December 2007.

[7] O. Volovikov, N. Kotilainen, T. Juonoja, M. Vapa, M. Weber, and J. Vuori, "Mobile encounter networks and their applications," in Proceedings of the 5th IEEE Consumer Communications and Networking Conference (CCNC '08), pp. 1176-1180, Las Vegas, Nev, USA, January 2008.

[8] W. J. Hsu and A. Helmy, "On nodal encounter patterns in wireless LAN traces," in Proceedings of the 2nd Workshop on Wireless Network Measurements (WiNMee '06), Boston, Mass, USA, April 2006.

[9] S. Tanachaiwiwat and A. Helmy, "On the performance evaluation and prediction of encounter-based worm interactions based on node characteristics," in Proceedings of the 2nd ACM Workshop on Challenged Networks (CHANTS '07), pp. 67-74, ACM, Montreal, Canada, September 2007.

[10] B. Walker, "Using persistent homology to recover spatial information from encounter traces," in Proceedings of the 9th International Symposium on Mobile Ad Hoc Networking and Computing (MobiHoc '08), pp. 371-380, ACM, Hong Kong, May 2008.

[11] G. Armstrong, P. Kotler, M. Harker, and R. Brennan, Marketing an Introduction, Pearson Educational, 2009.

[12] P. Kotler, G. Armstrong, and V. Wong, Principles of Marketing: European Edition, Prentice-Hall, Englewood Cliffs, NJ, USA, 1996.

[13] K. Mooney and N. Rollins, The Open Brand, AIGA Design Press, 2008.

[14] K. L. Keller, Strategic Brand Management. Building, Measuring and Managing Brand Equity, Prentice-Hall, Englewood Cliffs, NJ, USA, 2008.

[15] K. Torning and H. Oinas-Kukkonen, "Persuasive system design: state of the art and future directions," in Proceedings of the 4th International Conference on Persuasive Technology (Persuasive '09), vol. 350, pp. 1-8, ACM, Claremont, Calif, USA, April 2009.

[16] M. Harjumaa, K. Segerståhl, and H. Oinas-Kukkonen, "Understanding persuasive software functionality in practice: a field trial of polar FT60," in Proceedings of the 4th International Conference on Persuasive Technology (Persuasive'09), vol. 350, pp. 1-9, ACM, Claremont, Calif, USA, April 2009.

[17] F. Boström, P. Nurmi, P. Floréen, et al., "Capricorn-an intelligent user interface for mobile widgets," in Proceedings of the 10th International Conference on Human-Computer Interaction with Mobile Devices and Services (MobileHCI '08), pp. 327-330, ACM, Amsterdam, The Netherlands, September 2008.

[18] R. Peppers and M. Rogers, Managing Customer Relationship: A Strategic Framework, John Wiley \& Sons, Hoboken, NJ, USA, 2004. 
[19] D. Scott, R. Sharp, A. Madhavapeddy, and E. Upton, "Using visual tags to bypass Bluetooth device discovery," ACM SIGMOBILE Mobile Computing and Communications Review, vol. 9, no. 1, pp. 41-53, 2005.

[20] S. Weiss, "Widgets \& mobile 2.0," in Proceedings of the 9th International Conference on Human Computer Interaction with Mobile Devices and Services (MobileHCI '07), vol. 309, p. 370, ACM, Singapore, September 2007.

[21] A. Geven, R. Sefelin, N. Höller, M. Tscheligi, and M. Mayer, "Always-on information: services and applications on the mobile desktop," in Proceedings of the 10th International Conference on Human-Computer Interaction with Mobile Devices and Services (MobileHCI '08), pp. 23-32, ACM, Amsterdam, The Netherlands, September 2008. 

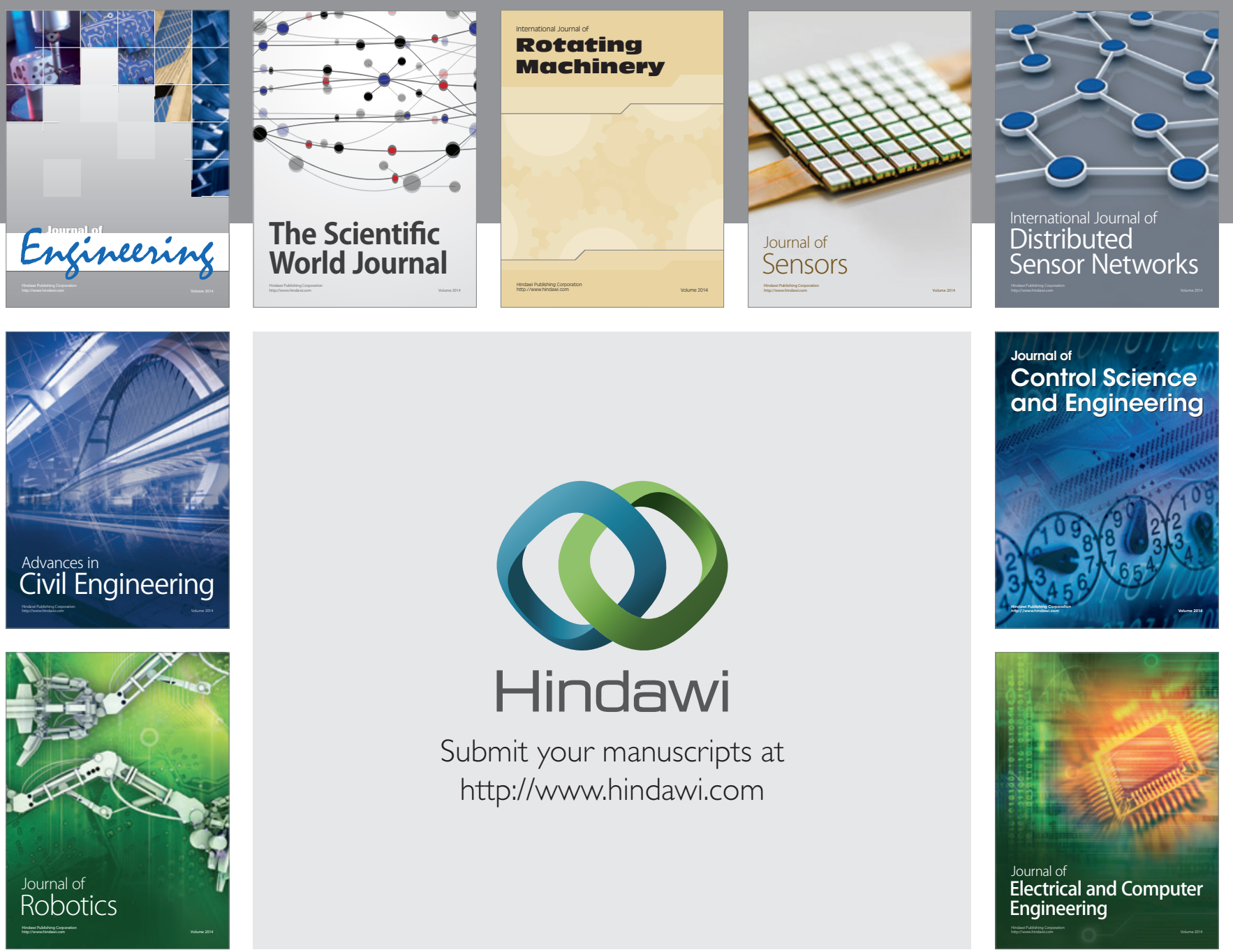

Submit your manuscripts at

http://www.hindawi.com
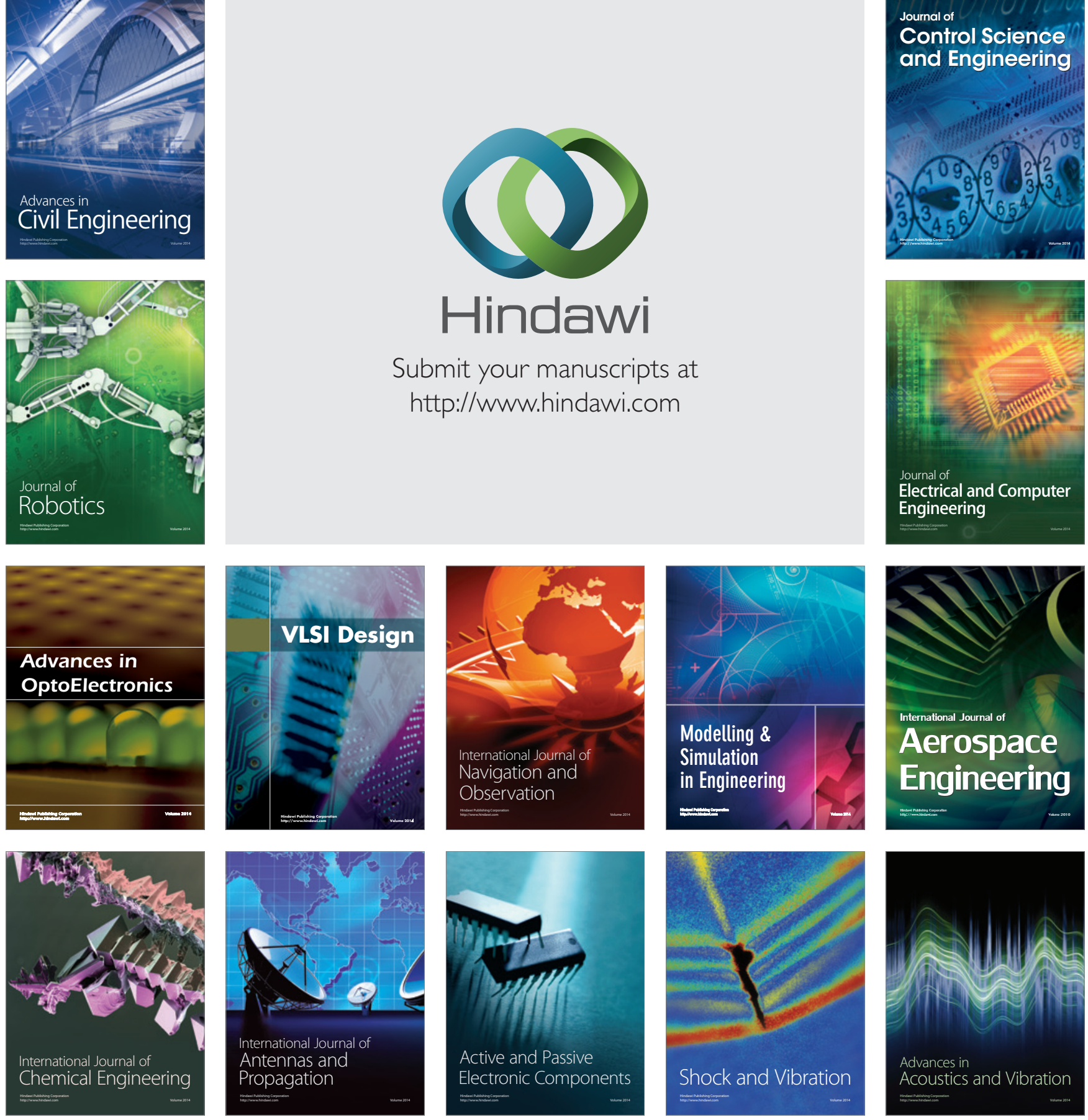